\title{
Los costos sociales de la contaminación hídrica en la microcuenca del río Las Cañas
}

\section{The social costs of water pollution in the microbasin of Las Cañas River}

\author{
José Ricardo Calles-Hernández \\ Investigador asociado de la Utec \\ ricalher@gmail.com
}

Recibido: 05/03/2015 - Aceptado: 20/06/2015

\section{Resumen}

El comportamiento de la morbilidad por IRC en la microcuenca del río Las Cañas se explica, al menos en un $50 \%$, por factores ambientales como la concentración de nitrógeno (nitrato y nitrito) en las fuentes de abastecimiento de agua para consumo humano y al caudal de vertido evidenciado en los municipios de Apopa, Soyapango y Tonacatepeque. No obstante, la relación entre las concentraciones de sustancias (químicas y orgánicas) y la morbilidad por insuficiencia renal crónica (IRC) es explicada, en un $95 \%$, cuando se asocian variables relacionadas con la edad y el consumo de agua de la población afectada. El costo social de la morbilidad por IRC, atribuible a la concentración de sustancias contaminantes identificadas en las fuentes de agua para abastecimiento para consumo humano analizadas en los municipios antes referidos equivale a US\$271.870.272.60 por año.

\section{Palabras clave}

Valoración económica, servicio ecosistémico, gestión hídrica.

\begin{abstract}
In at least $50 \%$ of cases, the ESRD morbidity in Las Cañas River microbasin is explained by environmental factors such as the concentration of nitrogen (nitrate and nitrite) in sources of water for human consumption, and the discharge rate evidenced in the municipalities of Apopa, Soyapango and Tonacatepeque.
\end{abstract}

However, the relationship between the concentrations of (chemical and organic) substances and end-stage renal disease (ESRD) morbidity is explained, $95 \%$ of the time, by the association of age-related variables and water consumption of the affected population. The social cost of ESRD morbidity attributable to the concentration of pollutants identified in water sources is equivalent to US $\$ 271.870 .272 .60$ per year.

\section{Keywords}

Economic assessment, ecosystemic service, water management. 


\section{Introducción}

Los recursos naturales del área metropolitana de San Salvador (AMSS) están sometidos a diferentes presiones antrópicas, como son el manejo inadecuado de desechos sólidos, el vertido de aguas residuales (sin previo tratamiento), la expansión de áreas para el desarrollo urbano en zonas ocupadas por ecosistemas de gran valor desde el punto de vista ecológico (bosque, cafetal y agro-ecosistemas), entre otras presiones.

Un territorio estratégico desde el punto de vista económico y comercial, localizado dentro del AMSS, es la microcuenca del río Las Cañas, ya que allí se realizan actividades industriales y comerciales bastante importantes a escala nacional; asimismo, posee un alto potencial agrológico y áreas con una buena capacidad de infiltración hídrica. En ella, aún se localizan espacios naturales con vegetación forestal que delimitan áreas en donde se desarrollan actividades agropecuarias, como la siembra de granos básicos y pastos naturales.

No obstante, las presiones antrópicas han generado efectos negativos sobre la calidad del agua en la microcuenca. En el 2011, el Ministerio de Medio Ambiente y Recursos Naturales (MARN) calculó un índice de calidad ambiental (y sanitaria) del agua que fluye sobre ese territorio, obteniendo como resultado una clasificación bajo la categoría de Pésima, es decir que "imposibilita el desarrollo de vida acuática", evidentemente debido al nivel de contaminación con sustancias tóxicas que presenta, entre ellas metales pesados.

Esta condición del recurso hídrico genera un costo social no cuantificado y mucho menos internalizado en las actividades productivas que se desarrollan en el territorio. Sin embargo, como primer paso en ese proceso es posible establecer, con la significancia estadística suficiente, la relación que existe entre la contaminación y la afectación de la salud humana en un territorio caracterizado por el desarrollo industrial, agropecuario y urbano.

En este artículo se demuestra que mediante un análisis dosis-respuesta es posible calcular ese costo social de la contaminación hídrica, lo cual podría ser útil para proponer algunas recomendaciones de control y regulación de la contaminación en la microcuenca, en el entendido de que el valor financiero calculado no representa un obstáculo para la gestión responsable y compartida del territorio y su capital natural y social. Por el contrario, el proceso metodológico de la investigación, así como los resultados que se obtengan, debe ponerse a disposición de los actores locales que hacen esfuerzos en la protección de la microcuenca, como una parte fundamental en una agenda de desarrollo para el territorio.

\section{La microcuenca del río Las Cañas}

Este sistema hídrico se localiza en los municipios de San Martín, Ilopango, Soyapango, Tonacatepeque, ciudad Delgado y Apopa (figura 1) y pertenece al departamento de San Salvador (figura 4.1). La elevación máxima es de $1.154 \mathrm{msnm}$, que corresponde a un punto alto del cerro de San Jacinto (Soyapango); y la mínima elevación es de 353 msnm, en el municipio de Apopa. Constituye un afluente del río Acelhuate que, a su vez, desemboca en el río Lempa, y posee una extensión de 7.503.5 hectáreas.

Figura. 1.

Ubicación de la microcuenca del río Las Cañas

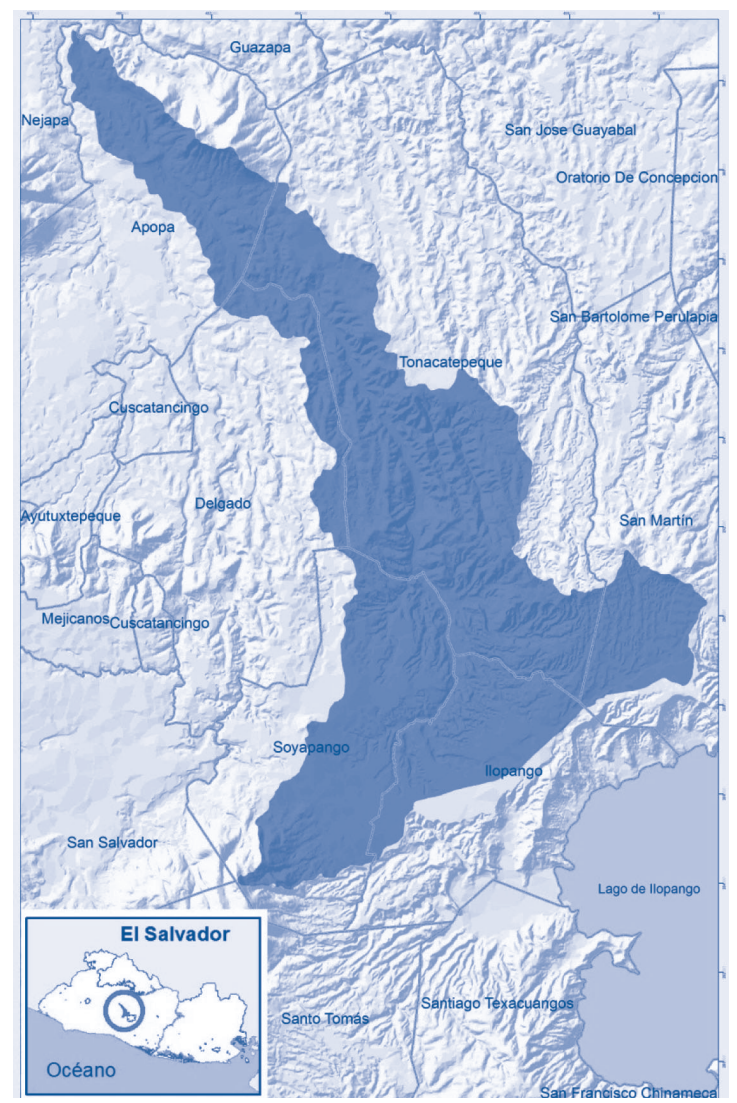


El recorrido principal del agua es de $21.5 \mathrm{~km}$ de longitud y desemboca en el río Acelhuate; tiene una dirección surestenoreste, aunque en su parte alta se dirige hacia el noreste. El cauce presenta una pendiente de 1,4\%. El sistema de drenajes sigue un patrón de tipo dendrítico en su dinámica fluvial, con la presencia de cursos de agua permanentes, intermitentes y efímeros.

Tomando en cuenta los resultados del Proyecto "Gestión intermunicipal de la cuenca del río Las Cañas bajo el enfoque de género y la reducción del riesgo de desastres El Salvador", se conoce la dirección del flujo de agua subterránea, el cual permite diferenciar tres zonas con distinto comportamiento hidrodinámico: (i) desde la parte media de la microcuenca hasta su desembocadura en el río Acelhuate, en donde el flujo subterráneo presenta una dirección sureste-noroeste, sentido noroeste, con dirección hacia el río, siendo este "receptor" y el acuífero "aportador"; (ii) margen izquierda del río, porción de los municipios de Delgado, soyapango e Ilopango, la cual constituye una vaguada en donde se aprecia una depresión en torno a la isopieza $520 \mathrm{msnm}$, lo cual puede ser explicado por la extracción de agua que se verifica en esos municipios; (iii) margen derecha del río, zona sur del municipio de Tonacatepeque y el de San Martín, en donde se observa la existencia de una divisoria de aguas subterráneas siguiendo una línea de dirección suroestenoreste.

\section{Caracterización de las aguas subterráneas}

Esta caracterización se basa en la distribución de la concentración de un parámetro químico en una determinada zona, a partir de la representación en círculos de distintos diámetros según la concentración en el punto de manera creciente, es decir, a mayor diámetro del círculo mayor será la concentración del parámetro. Para tal fin se ha echado mano a mapas de concentraciones, tomando en cuenta la campaña de muestreo realizada por el Proyecto "Gestión intermunicipal de la cuenca del río Las Cañas bajo el enfoque de género y la reducción del riesgo de desastres El Salvador" (2014).

\section{Figura 2.}

Red de muestreo comparada con la norma salvadoreña

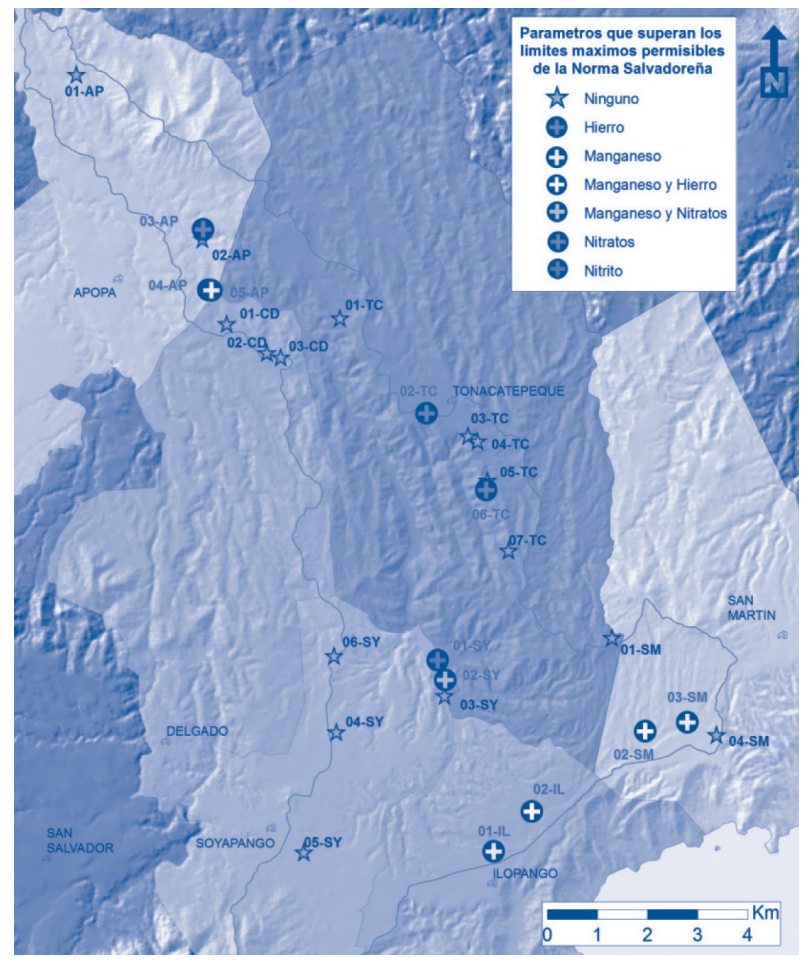

Fuente: Resultados del Proyecto "Gestión intermunicipal de la cuenca del río Las Cañas bajo el enfoque de género y la reducción del riesgo de desastres El Salvador" (2014).

Tanto el nitrito $\left(\mathrm{NO}_{2}\right)$, nitrato $\left(\mathrm{NO}_{3}\right)$, el hierro $(\mathrm{Fe})$ y el manganeso (Mn) son los parámetros químicos cuyas concentraciones superan el máximo permisible de acuerdo con la norma salvadoreña; no así el pH, el oxígeno disuelto, los cloruros, los sulfatos, el sodio, el calcio, el magnesio, el potasio, los fosfatos y el aluminio. En la figura 2 se muestra la distribución geográfica de los parámetros cuyas concentraciones han superado los límites permisibles. Cabe señalar que, por tratarse de resultados para una sola campaña de muestreo, se requiere cierta cautela a la hora de interpretar dichos parámetros.

Si se hace una comparación espacial, es posible encontrar una semejanza. Tal es el caso de los metales pesados hierro y manganeso, ya que sus concentraciones son superadas en tres municipios: Apopa, llopango y San Martín, precisamente los que presentan un gran desarrollo industrial. Sin embargo, el hierro también se supera en Tonacatepeque, donde no se da un desarrollo industrial importante; en cambio, sí se verifican actividades agropecuarias intensivas, que podrían 
ser causa de la presencia de nitrato por encima de la norma salvadoreña. Los puntos donde no se cumple la norma por la concentración de nitratos (Soyapango y Apopa) y nitritos (Soyapango) podrían estar influenciados por su cercanía al río Las Cañas y por la cercanía de fosas sépticas, ya que estos puntos constituyen pozos artesanales situados en colonias aledañas de su cauce. En la tabla 1 se presentan, marcados, los valores que superan los límites máximos permisibles, además se indica el uso al que se destina el agua y el municipio correspondiente.

Tabla 1.

Puntos de la red de muestreo que no cumplen la norma salvadoreña

\begin{tabular}{|c|c|c|c|c|c|}
\hline \multirow{4}{*}{ Municipio } & \multirow{4}{*}{ Uso } & \multicolumn{4}{|c|}{$\begin{array}{l}\text { Máximo permisible de acuerdo con la norma } \\
\text { salvadoreña }\end{array}$} \\
\hline & & 1.00 & 45.00 & 0.30 & 0.10 \\
\hline & & $\mathrm{mg} / \mathrm{l}$ & $\mathrm{mg} / \mathrm{l}$ & $\mathrm{mg} / \mathrm{l}$ & $\mathrm{mg} / \mathrm{l}$ \\
\hline & & $\mathrm{NO}_{2}$ & $\mathrm{NO}_{3}$ & $\mathrm{Fe}$ & $\mathrm{Mn}$ \\
\hline Apopa & Doméstico industrial sin consumo & - & 1.8360 & 0.8136 & 0.0599 \\
\hline Apopa & Doméstico familiar sin consumo & 0.1600 & 155.3570 & 0.0530 & 0.0120 \\
\hline Apopa & Doméstico familiar & 0.1640 & 1.0470 & 0.0737 & 3.1032 \\
\hline Delgado & Doméstico & - & - & 0.1500 & 0.2500 \\
\hline Tonacatepeque & Sin uso & 0.1290 & 136,5690 & 0.0630 & 0.0142 \\
\hline Soyapango & Sin uso & 1.5500 & 16.8400 & 0.0600 & 0.0118 \\
\hline Soyapango & Doméstico familiar sin consumo & - & 113.1260 & 0.1050 & 0.5028 \\
\hline Ilopango & Doméstico industrial sin consumo & 0.5350 & 1.1290 & 0.2096 & 1.0750 \\
\hline Ilopango & Doméstico industrial sin consumo & - & 0.8960 & 0.5642 & 0.5343 \\
\hline San Martín & Doméstico comunal & 0.2030 & 0.5860 & 0.3011 & 0.6851 \\
\hline San Martín & Doméstico comunal & - & 1.1050 & 0.0740 & 0.7312 \\
\hline
\end{tabular}

Fuente: Resultados del Proyecto "Gestión intermunicipal de la cuenca del río Las Cañas bajo el enfoque de género y la reducción del riesgo de desastres El Salvador" (2014).

Esta información permite conocer que, de los once puntos con valores no permitidos, en ocho se destina el agua para uso doméstico, de los cuales tres abastecen a familias, dos a comunidades y tres a fábricas o clubes sociales. Cabe señalar que la población que hace uso de estas fuentes de agua no tiene conocimiento de su calidad, y, por lo tanto, no están evaluando las repercusiones sobre la salud que conlleva su consumo. Aunque no es posible hablar de contaminación de la calidad de las aguas subterráneas, debido a que solo se dispone de información provista por una sola campaña de monitoreo, se sospecha de problemas puntuales con respecto al nitrito, nitrato, hierro y manganeso.

\section{Fuentes puntuales de contaminación del agua}

Según el MARN (2011), en la microcuenca se registran 19 vertidos: 11 provenientes de aguas residuales ordinarias y 8 de aguas residuales especiales. En la tabla 2 se muestran los detalles correspondientes a las fuentes puntuales de contaminación del agua. 
Tabla 2.

Fuentes fijas de contaminación hídrica

\begin{tabular}{|c|c|c|c|}
\hline Nombre & Tipo de vertido & Municipio & Cuerpo receptor \\
\hline Colector de aguas servidas Bosque de La paz & Especial & Ilopango & Quebrada Santa Lucía \\
\hline Aguas residuales de Cumbres de San Bartolo I y II & Ordinaria & Tonacatepeque & Río Las Cañas \\
\hline Descarga de aguas residuales de Residencial Libertad & Ordinaria & Tonacatepeque & Río Las Cañas \\
\hline Aguas residuales de colonia Sta. Teresa de Las Flores & Ordinaria & Apopa & Río Las Cañas \\
\hline Aguas residuales de la Urb. La Campanera & Ordinaria & Soyapango & Río La Campanera \\
\hline Aguas residuales de Rpto. San José 2 & Ordinaria & Soyapango & Tributario del río El Sauce \\
\hline Aguas residuales de Rpto. San José 3 & Ordinaria & Soyapango & Tributario del río El Sauce \\
\hline Aguas residuales de Urb. Brisas del Norte & Ordinaria & Tonacatepeque & Quebrada La Leona \\
\hline Aguas residuales de planta de tratamiento 1 , Urb. Altavista & Ordinaria & llopango & Quebrada Las Pavas \\
\hline Aguas residuales de planta de tratamiento 2, Urb. Altavista & Ordinaria & llopango & Quebrada Las Pavas \\
\hline Aguas residuales de colonia Cima de San Bartolo & Ordinaria & Tonacatepeque & Quebrada Las Pavas \\
\hline Aguas residuales de Reparto Las Cañas & Ordinaria & llopango & Río Las Cañas \\
\hline Colector de Bosques del Río & Especial & Soyapango & Río Las Cañas \\
\hline Aguas residuales de parque industrial El Desarrollo & Especial & Soyapango & Río Las Cañas \\
\hline Aguas residuales de sector Prusia & Especial & Soyapango & Río Las Cañas \\
\hline Aguas residuales de zona suroriente de Soyapango & Especial & Soyapango & Río Sumpa \\
\hline Aguas residuales colector 1, Zona Franca San Bartolo & Especial & Ilopango & Quebrada Arenal Seco \\
\hline Aguas residuales colector 2, Zona Franca San Bartolo & Especial & llopango & Quebrada Arenal Seco \\
\hline Aguas residuales colector 3, Zona Franca San Bartolo & Especial & llopango & Quebrada Arenal Seco \\
\hline
\end{tabular}

Fuente: MARN, 2011.

Este tipo de fuentes de contaminación son consideradas como descargas de agentes contaminantes (contenidos principalmente en aguas residuales ordinarias ${ }^{2}$ o especiales ${ }^{3}$ ), las cuales tienen una localización geográfica específica y que se realizan mediante una tubería hacia un cuerpo receptor, que puede ser el río Las Cañas o un tributario (quebrada). También se debe tener claro que en la zona rural de la microcuenca ocurren descargas particulares desde viviendas o comunidades situadas en las proximidades de los cuerpos receptores antes mencionados, las cuales son muy difíciles de cuantificar (dado su volumen y frecuencia intermitente), pero que en conjunto generan un impacto considerable.

\section{Fuentes no puntuales de contaminación del agua}

La disposición inadecuada de desechos sólidos y líquidos (aceites y lubricantes de motores) y la extracción de pétreos que se verifica en el cauce del río constituyen las fuentes no puntuales de contaminación del agua en el territorio de la microcuenca. En la tabla 3 se presentan los detalles correspondientes a estas fuentes.

\footnotetext{
2 Cuyo origen es la actividad doméstica.
} 
Tabla 3.

Fuentes no puntuales de contaminación hídrica

\begin{tabular}{lccc}
\multicolumn{1}{c}{ Nombre } & Cantón/Colonia & Municipio & Cuerpo receptor \\
Botadero a cielo abierto & Prusia & Soyapango & Río Las Cañas \\
Taller mecánico & Prusia & llopango & Río Las Cañas \\
Botadero a cielo abierto & Comunidad Sta. Rosa & Soyapango & Río Las Cañas \\
Botadero a cielo abierto & Reparto Las Cañas & llopango & Río Las Cañas \\
Botadero a cielo abierto & Cima de San Bartolo 1 & llopango & Quebrada Las Pavas \\
Botadero a cielo abierto & Cima de San Bartolo 2 & Tonacatepeque & Quebrada Las Pavas \\
Botadero a cielo abierto & Cumbres de San Bartolo & Tonacatepeque & Quebrada Las Pavas \\
Botadero de textiles y otros & Cantón Prusia & Soyapango & Río Sumpa \\
Botadero a cielo abierto & Comidad 3 de Enero, sector B & Soyapango & Río Las Cañas \\
Botadero a cielo abierto & Bosques del Río & Soyapango & Río Las Cañas \\
Botadero a cielo abierto & Cantón Joya Grande & Apopa & Río Las Cañas \\
Botadero de textiles & Lotificación Galindo & Apopa & Río Las Cañas \\
Botadero a cielo abierto & Cantón San Bartolo & Ilopango & Quebrada Arenal Seco \\
Extracción de arena & Arenal & Soyapango & Río Las Cañas \\
Fuente: MARN, 2011. & & &
\end{tabular}

\section{Uso del suelo en la microcuenca}

Con base en el mapa de uso actual del suelo, elaborado por la Universidad de El Salvador y la Fundación Salvadoreña para la Investigación del Café (Procafe) en el año 2010 (escala 1: 50.000), es posible establecer que la principal ocupación de las tierras es la actividad agropecuaria, que en su conjunto se desarrolla en el 53 \% de la microcuenca, lo que equivale a 3.964.27 ha; es decir, más de la mitad de la superficie del territorio. Esta ocupación corresponde a mosaico de cultivos y pastos, con el $27 \%$ de la superficie territorial (2.031.09 ha); seguido del $13 \%$ de terrenos principalmente agrícolas pero con importantes espacios de vegetación natural (equivalente a 984.51 ha); el $10 \%$ de áreas cultivadas con granos básicos (754.06 ha); el 2 \% de cultivos anuales asociados con otros cultivos (130.03 ha), y el $1 \%$ de pastos naturales (es decir, 64.58 ha). La tabla 4 muestra esta información. 
Tabla 4.

Uso del suelo

\begin{tabular}{|c|c|c|c|}
\hline Usos del suelo & & $\mathrm{Ha}$ & $\%$ \\
\hline Tejido urbano continuo & & 2.485 .56 & 33 \\
\hline Mosaico de cultivos y pastos & & 2.031 .09 & 27 \\
\hline Terrenos principalmente agrícolas & & 984.51 & 13 \\
\hline Granos básicos & & 754.06 & 10 \\
\hline Café & & 388.14 & 5 \\
\hline Arenales & & 226.5 & 3 \\
\hline Cultivos anuales asociados a otros cultivos & & 130.03 & 2 \\
\hline Tejido urbano discontinuo & & 126.41 & 2 \\
\hline Tejido urbano precario & & 124.47 & 2 \\
\hline Vegetación arbustiva baja & & 73.67 & 1 \\
\hline Vegetación herbácea natural & & 68.8 & 1 \\
\hline Pastos naturales & & 64.58 & 1 \\
\hline \multirow[t]{2}{*}{ Aeropuertos } & & 45.41 & 1 \\
\hline & Total & 7.503 .2 & \\
\hline
\end{tabular}

Asimismo, en el $33 \%$ predomina el tejido urbano continuo que, si se suma a las categorías tejido urbano discontinuo (2\%) y tejido urbano precario (2\%), entonces este porcentaje alcanza el valor de $37 \%$ como cobertura urbana (es decir 2.736.44 ha). La siguiente cobertura de importancia es el cultivo de café, con el $5 \%$, es decir, 388.14 hectáreas.

Se conoce que la microcuenca presenta extensas áreas de recarga hídrica, sobre todo en el municipio de Tonacatepeque y al noreste de ciudad Delgado, en donde los suelos están ocupados por actividades principalmente agrícolas. En ambos sitios, la capacidad de recarga asciende a $455 \mathrm{~mm}$ por año. En similar condición se encuentran las zonas de mosaicos de cultivos y pastos y de cultivos anuales en los alrededores del área urbana de San Martín, cuya capacidad de recarga se registra hasta en $475 \mathrm{~mm}$ anuales. Al noreste de la ciudad de Soyapango, también se pueden encontrar áreas ocupadas por cultivos y pastos, y con una capacidad de recarga que puede alcanzar los $335 \mathrm{~mm}$ por año.
La vulnerabilidad del acuífero se puede expresar en términos del riesgo que supone el tipo de actividad agropecuaria que se practica en la microcuenca y su entorno, ya que se trata de sistemas productivos con uso excesivo de agroquímicos, los cuales tienen la capacidad de penetrar gradualmente las capas del suelo y eventualmente llegar a contaminar el manto freático y al mismo acuífero. Un agroquímico utilizado frecuentemente es el fertilizante a base de nitrógeno, cuya absorción por parte de los cultivos es del 50 \%, de manera que el resto se lixivia y puede filtrarse hasta el acuífero. Esto posiblemente explica la concentración de nitratos, que superan (en algunos casos por más de tres veces) el límite permisible de la NSO 13.07.01:08 en Tonacatepeque (136.5690 mg/l), Soyapango (113.1260 mg/l) y Apopa $(155.3570 \mathrm{mg} / \mathrm{l})$, en donde efectivamente se tiene una ocupación del suelo netamente agropecuaria.

Por otra parte, también es relevante la comparación entre el uso del suelo urbano presente en la cabecera de la microcuenca, principalmente en los municipios de San 
Martín, llopango y Soyapango (siendo en estos dos últimos en donde se concentra una importante actividad industrial y comercial), con las concentraciones de hierro, manganeso y nitritos que sobrepasan los límites permisibles de la NSO 13.07.01:08, sobre todo, como se muestra en la tabla 4.1. Llama la atención la concentración de manganeso en Apopa (pozo de uso doméstico familiar), registrada en $3.1032 \mathrm{mg} / \mathrm{l}$, ya que representa un valor que supera en 31 veces el límite permitido por la NSO $(0.10 \mathrm{mg} / \mathrm{l})$; seguido del valor en llopango (pozo doméstico industrial sin consumo), de $1.0750 \mathrm{mg} / \mathrm{l}$, lo que equivale a casi once veces dicho límite.

\section{Población}

En 2014, la población de la microcuenca fue de 1.015.716 habitantes; de los cuales 378.072 corresponden a Soyapango (37\%); 166.069 a Apopa (16\%); 128.673 .55 a ciudad Delgado (13 \%); 124.624 a Ilopango (12 \%); 124.849.76 a Tonacatepeque (12 \%); y 93.428 a San Martín (9\%). De acuerdo con los datos de la tabla 5 y asumiendo un estimado de seis habitantes por vivienda, se tiene que en el territorio habrían 169.286 viviendas; 63.012 de ellas solo en Soyapango; 27.678 en Apopa; 20.771 en llopango; 21.446 en Tonacatepeque; 21.446 en Delgado; y 15.575 en San Martín.

Tabla 5.

Población por municipio y cantón en la microcuenca del río Las Cañas

\begin{tabular}{|c|c|c|c|}
\hline Municipio & Población & Viviendas & Porcentaje \\
\hline Apopa & 166.069 & 27.678 & 16 \\
\hline Ilopango & 124.624 & 20.771 & 12 \\
\hline San Martín & 93.428 & 15.571 & 9 \\
\hline Soyapango & 378.072 & 63.012 & 37 \\
\hline Tonacatepeque & 124.850 & 20.808 & 12 \\
\hline Delgado & 128.674 & 21.446 & 13 \\
\hline Total & 1.015 .717 & 169.286 & \\
\hline
\end{tabular}

Fuente: Elaboración propia a partir de datos catastrales municipales.

\section{Abastecimiento de agua potable}

Mayoritariamente, en la microcuenca se localizan sistemas de producción de agua potable que son administrados por la Administración Nacional de Acueductos y Alcantarillados (Anda) y operadores descentralizados, que en conjunto forman parte del Sistema Tradicional y Las Pavas de producción de Anda. La producción anual de agua en estos sistemas se muestra en la tabla 6. El consumo promedio mensual registrado en estos sistemas del territorio oscila entre 17 y $22 \mathrm{~m}^{3}$. En la región metropolitana de San Salvador, el consumo promedio es de $24 \mathrm{~m}^{3}$ (ver tabla 6). 
Tabla 6.

Producción de agua por municipios para el año $2011^{4}$

\begin{tabular}{c|r|r|}
\hline Municipio & Sistema & \multicolumn{1}{c}{$\begin{array}{c}\text { Producción } \\
\text { (en miles de } \mathrm{m}^{3} \text { ) }\end{array}$} \\
\hline Delgado & Tradicional & 8.333 .0 \\
Soyapango & Tradicional & 16.893 .0 \\
& Las Pavas & 7.885 .3 \\
Ilopango & Tradicional & 4.848 .3 \\
& Las Pavas & 2.628 .4 \\
San Martín & Tradicional & 605.9 \\
& Las Pavas & 2.553 .3 \\
Apopa & Tradicional & 12.878 .0 \\
& & $\mathbf{5 6 . 6 2 5 . 2}$
\end{tabular}

\section{Otras fuentes de abastecimiento de agua}

Con base en información del Proyecto "Gestión intermunicipal de la cuenca del río Las Cañas bajo el enfoque de género y la reducción del riesgo de desastres El Salvador" (2014), en la microcuenca existen otras fuentes de abastecimiento de agua (en uso), entre las que destacan 10 pozos artesanales, 10 manantiales de agua y 21 pozos con bombeo (tabla 7). Los pozos artesanales pertenecen a particulares que consumen en promedio un volumen de $14.5 \mathrm{~m}^{3}$ de agua al mes, siendo el abastecimiento doméstico su principal uso. Mientras que de los nacimientos de agua se consumen en promedio 1.5 m3 mensuales, para abastecimiento doméstico; en tanto que los pozos con bombeo se utilizan para el mismo fin, reportando un consumo promedio de $16 \mathrm{~m}^{3}$ al mes; y para abastecimiento industrial, del cual no se tiene registro mensual.

${ }^{4}$ La producción de agua es a través de los sistemas administrados por Anda y operadores descentralizados. 
Tabla 7.

Otras fuentes de abastecimiento de agua

\begin{tabular}{lccc}
\multicolumn{1}{c}{ Municipio } & $\begin{array}{c}\text { Pozos } \\
\text { artesanales }\end{array}$ & Nacimientos & $\begin{array}{c}\text { Pozos } \\
\text { con bombeo }\end{array}$ \\
Delgado & 3 & 2 & 2 \\
Soyapango & 5 & 2 & 5 \\
llopango & - & - & 6 \\
San Martín & - & 3 & 5 \\
Apopa & 2 & - & 3 \\
Tonacatepeque & - & 3 & -
\end{tabular}

Fuente: Elaboración propia con base en resultados del Proyecto "Gestión intermunicipal de la cuenca del río Las Cañas bajo el enfoque de género y la reducción del riesgo de desastres El Salvador" (2014).

De algunas de estas fuentes de abastecimiento se sabe que las concentraciones de nitrito, nitrato, hierro y manganeso superan los límites permisibles de la norma salvadoreña obligatoria para agua potable (NSO 13.07.01:08). En la tabla 8 se presenta información de la calidad de agua de los pozos y nacimientos de agua. En Apopa uno de los tres pozos con bombeo presenta una concentración de hierro, cuyo valor está casi tres veces por encima del límite permitido por la NSO, mientras que los dos pozos artesanales registrados en el proyecto antes referido también presentan problemas de calidad, ya que la concentración de nitrato de uno de ellos supera en tres veces el límite permisible establecido por la NSO; mientras que el otro posee una concentración de manganeso que supera el límite permitido por la norma en 31 veces.

En ciudad Delgado, uno de los dos pozos con bombeo presenta una concentración de manganeso que duplica el límite permisible. Mientras que en Soyapango, uno de los cinco pozos artesanales presenta una concentración de nitrato que supera el límite permisible en dos y media veces, mientras que el manganeso posee una concentración cinco veces superior al límite de la norma. Entre tanto, dos de los seis pozos con bombeo que existen en Ilopango tienen problemas de calidad debido a las concentraciones de hierro y manganeso; en uno de ellos, el manganeso registra una concentración que supera en casi once veces el valor del límite permitido por la NSO; en el otro, el hierro duplica el límite de la norma, y el manganeso lo quintuplica.

En San Martín el panorama es similar, ya que dos de los cinco pozos con bombeo presentan concentraciones de hierro y manganeso que superan la NSO. En ambos, el manganeso presenta una concentración siete veces mayor que la permitida en la norma; mientras que en uno de ellos, aunque con una mínima diferencia, el hierro supera el límite permisible por la NSO (tabla 4.8). 
Tabla 8.

Calidad de agua de otras fuentes de abastecimiento de agua

\begin{tabular}{|c|c|c|c|c|c|c|}
\hline \multirow{4}{*}{ Municipio } & \multirow{4}{*}{ Tipo } & \multirow{4}{*}{ Uso } & \multicolumn{4}{|c|}{$\begin{array}{c}\text { Máximo permisible de acuerdo con la norma } \\
\text { salvadoreña } \\
13.07 .01: 08\end{array}$} \\
\hline & & & 1,00 & 45,00 & 0,30 & 0,10 \\
\hline & & & $\mathrm{mg} / \mathrm{l}$ & $\mathrm{mg} / \mathrm{l}$ & $\mathrm{mg} / \mathrm{l}$ & $\mathrm{mg} / \mathrm{l}$ \\
\hline & & & $\mathrm{NO}_{2}$ & $\mathrm{NO}_{3}$ & $\mathrm{Fe}$ & $\mathrm{Mn}$ \\
\hline Арора & Pozo con bombeo & Doméstico industrial sin consumo & - & 1.84 & 0.81 & 0.06 \\
\hline Арора & Pozo artesanal & Doméstico familiar sin consumo & 0.16 & 155.36 & 0.05 & 0.01 \\
\hline Арора & Pozo artesanal & Doméstico familiar & 0.16 & 1.05 & 0.07 & 3.10 \\
\hline Delgado & Pozo con bombeo & Doméstico & - & - & 0.15 & 0.25 \\
\hline Soyapango & Pozo artesanal & Doméstico familiar sin consumo & - & 113.13 & 0.11 & 0.50 \\
\hline Soyapango & Pozo artesanal & Sin uso & 1.55 & 16.84 & 0.06 & 0.01 \\
\hline Ilopango & Pozo con bombeo & Doméstico industrial sin consumo & 0.54 & 1.13 & 0.21 & 1.08 \\
\hline Ilopango & Pozo con bombeo & Doméstico industrial sin consumo & - & 0.90 & 0.56 & 0.53 \\
\hline San Martín & Pozo con bombeo & Doméstico comunal & 0.20 & 0.59 & 0.301 & 0.69 \\
\hline San Martín & Pozo con bombeo & Doméstico comunal & - & 1.11 & 0.07 & 0.73 \\
\hline Tonacatepeque & Pozo artesanal & Sin uso & 0.13 & 136.57 & 0.06 & 0.01 \\
\hline
\end{tabular}

Puede observarse en la tabla anterior que en Soyapango se registra un pozo artesanal sin uso con una concentración de nitrito que supera el límite de la norma salvadoreña; y en la misma situación se encuentra un pozo en Tonacatepeque, cuya concentración de nitrato triplica el valor permisible de la NSO.

\section{Morbilidad por insuficiencia renal crónica}

A continuación se muestran los casos de morbilidad por insuficiencia renal agua (IRA) e IRC para el período comprendido entre 2006 y 2014, únicamente para Apopa, Soyapango y Tonacatepeque, ya que son estos municipios en los que se dispone de registros sobre abastecimiento humano con fuentes de agua subterránea que no cumple con los límites permisibles establecidos por la norma salvadoreña obligatoria 13.07.01:08, específicamente en los parámetros relacionados con la concentración de hierro, manganeso, nitrato y nitrito.

De acuerdo con los datos de la tabla 9, la IRA es menos frecuente que la IRC en los tres municipios analizados, ya que en un período de nueve años se reportan 217 casos de aquella (respecto a 8.064 casos de IRC) en el sistema de salud pública, siendo Soyapango el que registra el mayor número de enfermos, con 130 casos; seguido de Apopa, con 55; y Tonacatepeque, con 32. 
Tabla 9.

Morbilidad por insuficiencia renal aguda y crónica

\begin{tabular}{|c|c|c|c|c|c|c|c|c|c|c|}
\hline \multirow{2}{*}{\multicolumn{11}{|c|}{$\begin{array}{l}\text { Tipo de morbilidad/municipio } \\
\text { Insuficiencia renal aguda }\end{array}$}} \\
\hline & & & & & & & & & & \\
\hline Apopa & 10 & 5 & 3 & 5 & 6 & 5 & 5 & 8 & 8 & 55 \\
\hline Soyapango & 15 & 28 & 6 & 7 & 11 & 15 & 17 & 18 & 13 & 130 \\
\hline Tonacatepeque & 3 & 2 & 2 & 2 & 3 & 2 & 8 & 5 & 5 & 32 \\
\hline Total & 28 & 35 & 11 & 14 & 20 & 22 & 30 & 31 & 26 & 217 \\
\hline \multicolumn{11}{|l|}{ Insuficiencia renal crónica } \\
\hline Apopa & 301 & 326 & 249 & 196 & 254 & 232 & 275 & 409 & 350 & 2.592 \\
\hline Soyapango & 409 & 422 & 386 & 384 & 480 & 520 & 495 & 760 & 587 & 4.443 \\
\hline Tonacatepeque & 31 & 87 & 84 & 91 & 77 & 109 & 145 & 204 & 201 & 1.029 \\
\hline Total & 741 & 835 & 719 & 671 & 811 & 861 & 915 & 1.373 & 1.138 & 8.064 \\
\hline
\end{tabular}

Fuente: Elaboración propia con base en Sistema de Morbi-mortalidad en línea SIMMOW, Códigos N17.0-N17.9 (Clasificación Internacional de Enfermedades y Problemas Relacionados con la Salud). *Dato a octubre de 2014.

Gráfico 1.

Morbilidad por insuficiencia renal aguda

- Apopa "Soyapango $\square$ Tonacatepeque

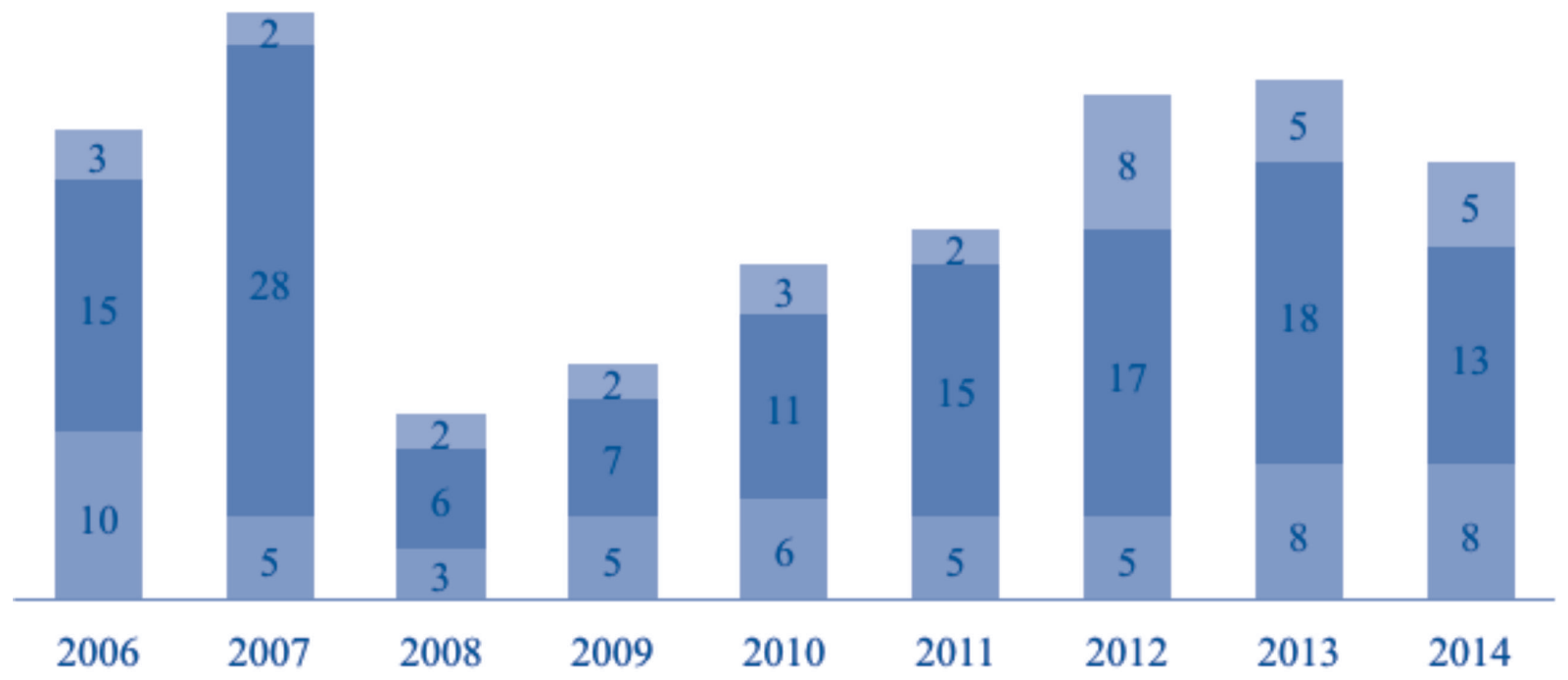

Fuente: Elaboración propia. 
El gráfico 1 permite observar un aumento gradual en Soyapango y Tonacatepeque en el período 2008-2013, pasando de 6 a 18 y de 2 a 5 casos, respectivamente. En Apopa la tendencia parece ser variable, aunque en ese mismo período se tiene un aumento de 3 a 8 casos reportados de IRA. Con relación a la IRC, es Soyapango el municipio que reporta más números de casos para el mismo período, 4.443 en total, verificando una tendencia al alza desde el año 2006 hasta el 2013, pasando de 301 casos a más del doble (760) en siete años.

Situación más crítica experimenta Tonacatepeque, que para el mismo período reporta un aumento de casi siete veces el número de casos de morbilidad por IRC. En Apopa se registran un mayor número de casos que Tonacatepeque
(2.592 contra 1.209), aunque el aumento no es tan marcado como en los otros dos municipios, ya que solo se observan 108 casos más entre 2006 y 2013.

En el análisis de la morbilidad por IRC (gráfico 2) es importante incluir la variable edad, ya que el comportamiento en el número de casos varía dependiendo del rango en el número de años de la población. En la tabla 4.15 se pueden observar estas variaciones por municipio para el período 2006-2014. De esta forma, se visualiza que del rango de 1 a 14 años el número de casos de morbilidad por IRC no presenta mayores variaciones en su frecuencia; no así cuando se trata del rango entre 15 y 60 años, para el que prácticamente se incrementan entre 10 y 20 veces.

Gráfico 2.

Morbilidad por insuficiencia renal crónica

- Apopa - Soyapango $\quad$ Tonacatepeque

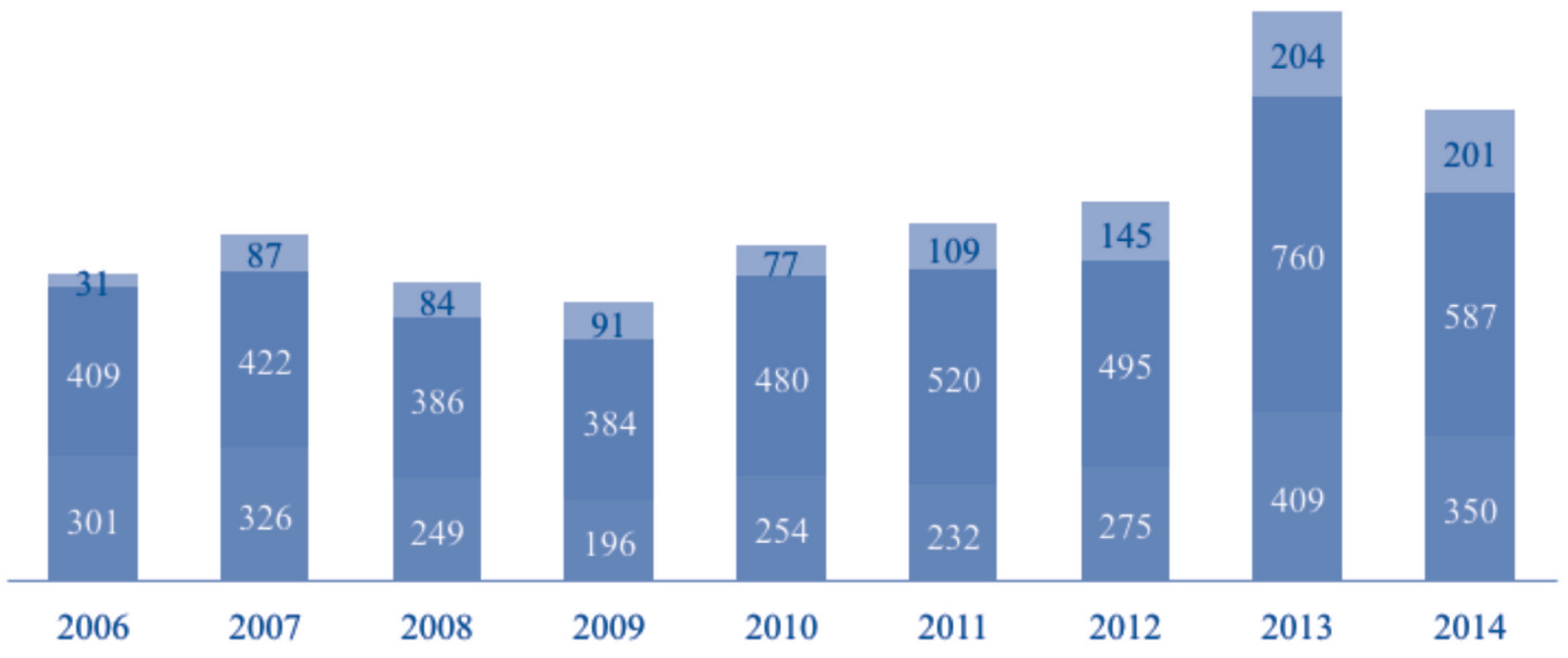

Fuente: elaboración propia. 
Tabla 10.

Morbilidad por insuficiencia renal crónica

(por rango de edades)

\begin{tabular}{|c|c|c|c|c|c|c|c|c|}
\hline \multirow{2}{*}{ Municipios } & \multirow{2}{*}{ Años } & \multicolumn{6}{|c|}{ Edades (años) } & \multirow{2}{*}{ Total } \\
\hline & & $1-4$ & $5-9$ & $10-14$ & $15-19$ & $20-59$ & $\geq 60$ & \\
\hline \multirow{9}{*}{ Apopa } & 2006 & 5 & 2 & 18 & 29 & 115 & 132 & 301 \\
\hline & 2007 & 4 & 3 & 17 & 34 & 146 & 122 & 326 \\
\hline & 2008 & 9 & 3 & 4 & 27 & 101 & 105 & 249 \\
\hline & 2009 & 5 & 1 & 4 & 11 & 87 & 88 & 196 \\
\hline & 2010 & 5 & 5 & 7 & 11 & 112 & 114 & 254 \\
\hline & 2011 & 1 & 6 & 4 & 9 & 112 & 100 & 232 \\
\hline & 2012 & 6 & 5 & 3 & 11 & 136 & 114 & 275 \\
\hline & 2013 & 4 & 4 & 3 & 9 & 194 & 195 & 409 \\
\hline & $2014^{*}$ & 2 & 3 & 6 & 8 & 162 & 169 & 350 \\
\hline \multirow{9}{*}{ Soyapango } & 2006 & 4 & 6 & 6 & 7 & 182 & 204 & 409 \\
\hline & 2007 & 14 & 11 & 9 & 12 & 199 & 177 & 422 \\
\hline & 2008 & 13 & 13 & 7 & 17 & 158 & 178 & 386 \\
\hline & 2009 & 10 & 1 & 6 & 15 & 187 & 165 & 384 \\
\hline & 2010 & 4 & 7 & 9 & 0 & 254 & 206 & 480 \\
\hline & 2011 & 2 & 9 & 8 & 20 & 260 & 221 & 520 \\
\hline & 2012 & 6 & 8 & 17 & 18 & 232 & 214 & 495 \\
\hline & 2013 & 8 & 9 & 48 & 28 & 349 & 318 & 760 \\
\hline & $2014^{*}$ & 8 & 11 & 12 & 17 & 268 & 271 & 587 \\
\hline \multirow{10}{*}{ Tonacatepeque } & 2006 & 0 & 0 & 5 & 0 & 26 & 0 & 31 \\
\hline & 2007 & 4 & 1 & 1 & 3 & 41 & 37 & 87 \\
\hline & 2008 & 1 & 0 & 6 & 3 & 35 & 39 & 84 \\
\hline & 2009 & 1 & 0 & 3 & 7 & 41 & 39 & 91 \\
\hline & 2010 & 1 & 1 & 1 & 3 & 27 & 44 & 77 \\
\hline & 2011 & 2 & 0 & 14 & 6 & 37 & 50 & 109 \\
\hline & 2012 & 1 & 3 & 7 & 3 & 79 & 52 & 145 \\
\hline & 2013 & 3 & 43 & 15 & 19 & 54 & 70 & 204 \\
\hline & $2014^{*}$ & 1 & 3 & 42 & 11 & 72 & 72 & 201 \\
\hline & Total & 124 & 158 & 282 & 338 & 3.666 & 3.496 & 8.064 \\
\hline
\end{tabular}

Fuente: Elaboración propia con base en Sistema de Morbi-mortalidad en línea SIMMOW, Códigos N17.0-N17.9 (Clasificación Internacional de Enfermedades y Problemas Relacionados con la Salud). *Dato a octubre de 2014. 
Esto ocurre en Soyapango desde el 2006, en donde se visualiza una diferencia amplia entre ambos rangos de edad y el número de casos reportados de IRC. En ese año, se registraban 7 casos (para el rango de 15-19 años) contra 182 (para las edades entre 20-59 años). Mientras que para 2014 se contabilizan 17 para el primer rango y 268 para el segundo. Lo mismo pasa en Apopa, en donde se puede observar que en 2013 el registro de casos aumentó de 9 (para el rango de 15-19 años) a 194 (para las edades entre 20-59 años); y para 2014, la frecuencia de casos pasó de 8 para el primer rango de edades, a 162 para el segundo. A pesar de que en Tonacatepeque no se presenta el mismo volumen de casos de morbilidad por IRC, como en Apopa y Soyapango, también es notoria la diferencia entre las frecuencias de un rango de edad a otra.

Es conveniente señalar que, entre los rangos de edad 20-29 $y \geq 60$, prácticamente no se perciben diferencias; aunque, al observar detenidamente, pareciera que los casos de morbilidad tienden a reducirse en edades superiores a los 60 años (tabla 10).

\section{Análisis dosis-respuesta para valorar el costo social de la contaminación hídrica}

El análisis dosis-respuesta está enfocado particularmente en la IRC, como un efecto derivado de la contaminación del agua en la salud de la población que reside en la microcuenca del río Las Cañas. Estimados entonces los niveles de deposición de sustancias contaminantes en los sistemas hídricos (dosis) de la mircrocuenca con el número casos de morbilidad (respuesta), para el período de 9 años: de 2006 a 2014, es posible establecer una función dosisrespuesta que mida el nivel de sensibilidad o elasticidad de la variable dependiente (daño en la salud) versus los cambios en la calidad de un recurso natural.

Se entenderá como elasticidad al cambio porcentual en la morbilidad, derivado del cambio en la concentración o volumen de contaminantes del agua $\left(\partial \mathrm{Morb} /\left(\partial Q_{\mathrm{v}}\right)\right.$; y al ser cuantificada permite relacionar el costo médico (precio de mercado) necesario para atender los efectos de la contaminación en la salud de una población determinada, en un espacio temporal y geográfico particular. Los costos médicos pueden incluir los de hospitalización, tratamiento o medicamentos (ISBM, 2013).

El análisis dosis-respuesta está basado en un modelo econométrico que toma en cuenta tres consideraciones fundamentales, que son las siguientes:

1. Solo se han tomado en cuenta los casos de IRC, entendida esta como la "pérdida gradual y progresiva de la capacidad de excretar los desechos nitrogenados, concentrar la orina y mantener la capacidad de homeostasis del medio interno por el riñón" (González Álvarez \& Mallafré Andruig, 2009), también considerada como uno de los estadios de la ERC.

2. La población de referencia son personas con rangos de edad que van desde los 10 hasta más de 60 años. Esto se propone tomando en consideración el comportamiento de los casos de morbilidad por IRC respecto a la edad (tabla 10), durante un período de nueve años, lo cual dejaría por fuera a las personas por debajo de 10 años de edad.

3. El objeto de estudio es la microcuenca del río Las Cañas, y sobre todo los municipios en donde se ha evidenciado que las fuentes de abastecimiento de agua para uso doméstico tienen problemas de calidad; sea por la concentración de hierro y manganeso o por los valores de nitritos y nitratos. En tal sentido, se hará referencia a Soyapango, Tonacatepeque y Apopa.

El análisis se basa en información anual del período 20062014 presentada en la tabla 11, en cuya segunda columna se observa el número de casos de IRC con edades que varían entre los 10 y 60 años (o más), registrados en el sistema público de salud, para los municipios que conforman la microcuenca del río Las Cañas, y en los que se localizan fuentes de agua para abastecimiento humano (pozos y manantiales), con afectaciones en su calidad hídrica. En la tercera columna se registra la población que tiene influencia sobre dichas fuentes de agua.

La cuarta columna es la serie de la tasa bruta de morbilidad por diez mil habitantes $(T B M=$ Morbilidad $\mathrm{x}$ 10.000/Población), ya que difícilmente se puede estimar 
una tasa específica, en tanto que no existe información confiable sobre el tamaño de la población en los rangos de edad ya señalados, y que residan específicamente dentro de los límites de la microcuenca. No obstante, es de esperarse cierta proporcionalidad entre ambas tasas. A partir de la quinta columna se muestran las concentraciones de las sustancias relacionadas con la calidad de las fuentes de agua establecidas previamente, tomando en cuenta el consumo mensual promedio de agua. En la octava columna se exhibe la serie de datos correspondiente al volumen de vertido anual (desde fuentes puntuales y no puntuales), estimados a partir de las cargas contaminantes que fluyen por el río Las Cañas y sus tributarios.

Tabla 11.

Variables del análisis dosis-respuesta

\begin{tabular}{|c|c|c|c|c|c|c|c|}
\hline Años & Morbilidad & Población & TBM & $\begin{array}{c}\mathrm{N} \\
\text { kg/año }\end{array}$ & $\begin{array}{c}P \\
\text { kg/año }\end{array}$ & $\begin{array}{c}\text { DQO } \\
\text { kg/año }\end{array}$ & $\begin{array}{c}\text { QV } \\
\mathrm{m}^{3} / \text { año }\end{array}$ \\
\hline 2006 & 741 & 332.057 & 22,32 & 83.348 & 37.625 & 1.571 .483 & 3.898 .418 \\
\hline 2007 & 835 & 341.023 & 24,49 & 76.402 & 34.490 & 1.440 .526 & 3.573 .549 \\
\hline 2008 & 719 & 345.878 & 20,79 & 78.361 & 35.374 & 1.477 .463 & 3.665 .179 \\
\hline 2009 & 671 & 351.650 & 19,08 & 129.915 & 58.647 & 2.449 .478 & 6.076 .481 \\
\hline 2010 & 811 & 331.617 & 24,46 & 569.627 & 257.143 & 10.740 .018 & 26.643 .032 \\
\hline 2011 & 861 & 336.191 & 25,61 & 1.396 .505 & 630.414 & 26.330 .368 & 65.318 .400 \\
\hline 2012 & 915 & 340.442 & 26,88 & 1.586 .937 & 716.380 & 29.920 .872 & 7.425 .455 \\
\hline 2013 & 1373 & 344.391 & 39,87 & 1.753 .983 & 791.788 & 33.070 .438 & 82.038 .660 \\
\hline 2014 & 1138 & 424.891 & 26,78 & 1.238 .106 & 558.909 & 23.343 .838 & 57.909 .643 \\
\hline
\end{tabular}

Fuente: Elaboración propia

El análisis dosis-respuesta considera que el volumen de vertido de sustancias tóxicas al cauce (y los tributarios) del río Las Cañas afecta la calidad de agua de las fuentes utilizadas para abastecimiento humano, evidenciado por la presencia de hierro, manganeso, nitrito y nitrado en concentraciones fuera de los límites permisibles de la NSO; por lo tanto, dicho vertido es considerado como una variable proxy de la contaminación hídrica, suponiendo proporcionalidad entre ambas variables.

Con el objetivo de logra una buena significancia estadística, la información sobre la morbilidad se descompone en dos subgrupos: personas menores de 20 años (o iguales) y mayores de 20 años. Esta desagregación hace que el número de observaciones independientes se duplique. Dicha segregación se presenta en la tabla 12. De esta forma, se utilizan los mínimos cuadrados ordinarios para correr una regresión del logaritmo natural de los casos de morbilidad por IRC, contra el logaritmo del volumen de vertidos (asociados a las sustancias químicas y orgánicas contaminantes), el logaritmo del consumo de agua y una variable dicótoma (dummy) de la edad que asume el valor 0 para personas menores de 20 años y 1 para personas mayores o iguales a 20 años. 
Tabla 12.

Morbilidad por insuficiencia renal crónica (con referencia de 20 años)

\begin{tabular}{|r|rrrrrrrrrr}
$\begin{array}{c}\text { Municipio/Año } \\
\text { Menores de 20 }\end{array}$ & 2006 & 2007 & 2008 & 2009 & 2010 & 2011 & 2012 & 2013 & 2014 \\
Apopa & 54 & 58 & 43 & 21 & 28 & 20 & 25 & 20 & 19 \\
Soyapango & 23 & 46 & 50 & 32 & 20 & 39 & 49 & 93 & 48 \\
Tonacatepeque & 5 & 9 & 10 & 11 & 6 & 22 & 14 & 80 & 57 \\
Total parcial & 82 & 113 & 103 & 64 & 54 & 81 & 88 & 193 & 124 \\
\hline Mayores de 20 & & & & & & & & & \\
Apopa & 247 & 268 & 206 & 175 & 226 & 212 & 250 & 389 & 331 \\
Soyapango & 386 & 376 & 336 & 352 & 460 & 481 & 446 & 667 & 539 \\
Tonacatepeque & 26 & 78 & 74 & 80 & 71 & 87 & 131 & 124 & 144 \\
\hline Total parcial & 659 & 722 & 616 & 607 & 757 & 780 & 827 & 1.180 & 1.014 \\
\hline Fuente: Elaboración propia & & & & & & & & &
\end{tabular}

A partir de un análisis de regresión se visualiza un ajuste bastante bueno $\left(\mathrm{R}^{2}=95 \%\right)$, y los coeficientes presentan los signos esperados, con niveles de significancia aceptables. En esas condiciones, la estimación de la elasticidad de la morbilidad ante el vertido de sustancias químicas y orgánicas al sistema hídrico es 0.425 (algunas unidades más elevado que la regresión 3). El signo positivo del coeficiente de la variable dummy (Edad_1) es positivo, lo cual parece indicar, como era de esperarse, que las personas mayores o iguales a 20 años son más propensas a mostrar las afectaciones características de la IRC, por efecto de las condiciones ambientales relacionadas con el recurso hídrico de la microcuenca. Finalmente, el modelo econométrico que mejor explica la relación entre la morbilidad por IRC y las variables relacionadas con la calidad del agua es el siguiente:

\section{Morbilidad por $\mathrm{I}=4,21+0,92($ Edad) $-0,425$ (Vertido) $+0,5$ (Consumo de agua)}

\section{Estimación del costo marginal de la contaminación hídrica}

Para lograr estimar este costo, el primer paso consiste en cuantificar la relación entre las variables caudal de vertido y las concentraciones de las sustancias químicas y orgánicas analizadas. Con base en los estudios de contaminación realizados en 2011 por el MARN, se parte del supuesto de que existe una relación proporcional entre los caudales de vertido (por fuentes puntuales principalmente) y las concentraciones de los parámetros de calidad de agua (metales pesados y nitrógeno). Asimismo, se puede suponer (a partir de lo análisis hidrogeoquímicos presentados anteriormente) que el uso del suelo, sobre todo por actividades agropecuarias (con uso intensivo de agroquímicos) e industriales, relacionado con sus características de textura y estructura (que le confieren mayor o menor capacidad de recarga hídrica), influyen directamente en la concentración de algunas sustancias contaminantes, como el hierro, manganeso, nitrito y nitrato.

La elasticidad de la morbilidad por $\mathrm{IRC}$, respecto a la concentración de sustancias contaminantes en los cuerpos de agua, es de 0.425 (según el modelo determinado anteriormente). Suponiendo que la elasticidad de la concentración con respecto a la emisión es cercana (o igual) a 1, entonces la elasticidad de la morbilidad con respecto 
a la concentración de sustancias contaminantes es igual a 0.425, como se presenta a continuación.

$$
\begin{aligned}
\text { Si } \frac{\partial M o r b}{\partial C} \times & \frac{C}{M o r b}=0.425 ; y \text { si } \frac{\partial C}{\partial Q_{v}} \times \frac{Q_{v}}{C} \\
& =1 ; \text { entonces, } \frac{\partial M o r b}{\partial Q_{v}} \times \frac{Q_{v}}{M o r b}=0.425
\end{aligned}
$$

La estimación más actual realizada en el cauce del río Las Cañas, respecto al vertido de sustancias contaminantes en el cauce y sus tributarios, es la que realizó el MARN en el marco del Programa Nacional de Reducción de Riesgo, a partir del estudio denominado "Medidas de control de la contaminación de los ríos Tomayate y Las Cañas", en 2011. En ese estudio registró un caudal de vertido de $2.1 \mathrm{~m}^{3} / \mathrm{s}$, el cual debe ser proporcional a las fuentes puntuales de contaminación que tienen más incidencia geográfica en los pozos y nacimientos de los que se abastece la población afectada. Entre tanto, para el período 2011-2014, el Minsal registra un total de 4.287 casos de morbilidad por IRC. Por lo cual, un estimado de la relación marginal entre morbilidad y caudal de vertido es 0.1 ( $\partial \mathrm{Morb} /\left(\partial Q_{\mathrm{V}}\right)$.

Si se sabe que el costo promedio anual del tratamiento total de un paciente con IRC es de U\$6.000, sin medicamentos (Instituto Salvadoreño de Bienestar Salvadoreño, 2011); y si a ese costo se le calcula el $25 \%$ por gastos en medicamentos, se tiene un costo anual de US\$7.500. De manera que si este costo promedio es aproximado al costo marginal (suponiendo un rendimiento decreciente del servicio de atención en salud, subvalorando el costo marginal), el costo social por el vertido de sustancias contaminantes al sistema hídrico de la microcuenca del río Las Cañas, con su consecuente afectación a los cuerpos receptores que son aprovechados para el abastecimiento de agua para consumo humano, es de aproximadamente US\$750.00 (de 2011), que equivale a US\$768.03 (de 2013), teniendo en cuenta el índice de precios industriales. De esta forma, si se considera la estimación del vertido de sustancias químicas y orgánicas registrado en el año 2011, se obtiene un costo total de US\$27.870.272.60 (de 2013) por año.

\section{Conclusión}

Tomando en cuenta que en la microcuenca del río Las Cañas existen algunas fuentes de agua utilizadas para el abastecimiento humano que presentan concentraciones de hierro, manganeso, nitrito y nitrato que sobrepasan (en varias veces) los límites permisibles de la NSO, y que estas se localizan en Apopa, Ilopango y San Martín, que son precisamente los municipios en donde se verifica una intensa actividad industrial y agropecuaria, es posible encontrar una relación con el número de casos de insuficiencia renal aguda, los cuales, en el lapso del 2006 al 2014, ascienden a 217, mientras que los de morbilidad por IRC suman 8.064 casos. Un detalle de esa relación es que entre los pacientes de 20 a 60 (o más) años se registra mayor morbilidad.

Con base en los análisis de regresión realizados, se puede afirmar que, al menos en un $50 \%$, esa relación se explica por factores ambientales como la concentración de nitrógeno (nitrato y nitrito) en las fuentes de abastecimiento de agua para consumo humano, y al caudal de vertido en los municipios de Apopa, Soyapango y Tonacatepeque. No obstante, la relación entre estas concentraciones de sustancias químicas y la morbilidad por IRC es explicada en un $95 \%$ cuando se asocian variables relacionadas con la edad y el consumo de agua de la población afectada. De forma matemática, esa relación se expresa de la siguiente manera:

Morbilidad por I=4,21+0,92(Edad)-0,425(Vertido) +0,5(Consumo de agua)

Este modelo permite establecer que la elasticidad de la morbilidad ante el vertido de sustancias químicas y orgánicas al sistema hídrico es 0.425 , es decir, que un incremento del $10 \%$ en el vertido de sustancias contaminantes aumenta Ios casos de morbilidad por IRC en $4 \%$. Asimismo, esta relación matemática permite confirmar que el costo social de la morbilidad por IRC, atribuible a la concentración de nitrógeno (nitrato y nitrito) en las fuentes de abastecimiento de agua para consumo humano y al caudal de vertido en los municipios de Apopa, Soyapango y Tonacatepeque, equivale a US\$27.870.272.60 (de 2013) por año. 


\section{Recomendaciones}

La depuración hídrica es urgente en la microcuenca del río Las Cañas; o, en su defecto, el control de la calidad del agua en las fuentes de abastecimiento humano (pozos y nacimientos), sobre todo en las que se les ha detectado una concentración mayor de la que establecen los límites permisibles de la norma salvadoreña obligatoria para agua potable. Esto debe ser así, ya que está demostrado que existe una relación entre la morbilidad por IRC y el consumo de agua con alteraciones en algunos de sus parámetros de calidad. Para ello, es fundamental la gestión compartida que puedan realizar los gobiernos locales y otros actores públicos y privados (incluidas las organizaciones de la sociedad civil), en virtud de asegurar un mayor cumplimiento ambiental por parte del sector industrial y del subsector de agua potable y saneamiento. Dicha gestión debe estar acompañada por la rectoría a la que está facultado ejercer el MARN respecto al cumplimiento de la normativa para el control de la contaminación hídrica.

Con base en los valores monetarios del costo social por contaminación del agua, es necesario crear los mecanismos financieros y fiscales correspondientes, para que los actores reconozcan el costo social por la contaminación hídrica en la microcuenca del río Las Cañas. Esto puede ser posible a través de la creación de un fondo de agua, constituido por aportaciones monetarias de organizaciones públicas y privadas, para ser invertidos en la conservación de la microcuenca (incluidas acciones de recuperación del recurso hídrico), llevando consigo beneficios sociales en términos de mejoras en la calidad de vida de la población. Este mecanismo financiero es capaz de fundarse sin un marco legal nacional del sector hídrico; por el contrario, puede ser el resultado de procesos de concertación y definición de acuerdos entre actores interesados en la protección del agua como recurso dinamizador del desarrollo territorial.

\section{Referencias}

Anda (2013). Boletín estadístico 2011. El Salvador.

ISBM (2013). Guías clínicas de atención a las personas con enfermedad renal crónica. San Salvador, El Salvador.

Ministerio de Medio Ambiente y Recursos Naturales (2011). "Medidas de Control de la Contaminación de los ríos Tomayate y Las Cañas". San Salvador, El Salvador: Programa Nacional de Reducción de Riesgo.

MARN (Jueves, 22 de julio de 2010). Ministerio de Medio Ambiente y Recursos Naturales. Recuperado el 1 de noviembre de 2014 de http://www.marn.gob.sv/ index. php?option=com_content\&view=article $\&$ id $=27$ 5\&ltemid=197

Minsal (2013). Boletín Epidemiológico, "Semana Epidemiológica 47". San Salvador. 